\title{
Choosing exercise or pharmacologic stress imaging, or exercise ECG testing alone: How to decide
}

\author{
Jorge A. Gonzalez, MD, ${ }^{a}$ and George A. Beller, $M D^{a}$ \\ a University of Virginia, Charlottesville, VA
}

Received Jan 10, 2016; accepted Jan 11, 2016

doi: 10.1007/s12350-016-0409-3

\section{See related article, pp. 546-554}

Cardiac imaging of either myocardial perfusion or regional function, under resting and stress conditions, utilizing either nuclear cardiology or ultrasound technology, is the dominant approach to the noninvasive detection of coronary artery disease (CAD) in symptomatic patients. Prior to the advent of imaging technology that permitted assessment of myocardial perfusion and function, symptom-limited exercise electrocardiography (ExECG) alone was the test employed for detecting CAD. Multiple clinical studies showed that combining imaging with symptomlimited exercise enhanced the diagnostic and prognostic values of exercise testing, over the variables derived from ExECG. Pharmacologic stress then emerged as an alternative to exercise for imaging of patients who were deemed unable to attain a target heart rate of $\geq 85 \%$ of maximum predicted heart rate adjusted for age. Submaximal exercise decreases the sensitivity of MPI for detection of ischemia and prevents accurate measurement of extent of ischemia when present. ${ }^{1}$

With either SPECT or PET myocardial perfusion imaging (MPI), intravenously administered vasodilators such as dipyridamole, adenosine, and regadenoson substituted as stressors for exercise. These agents allowed for detection of relative flow reserve alterations between stenotic and normal coronary arteries. Dobutamine infusion was reserved for patients with bronchospasm, or those who ingested caffeine prior to testing. For stress echocardiography, dobutamine

Reprint requests: Jorge A. Gonzalez, MD, University of Virginia, Charlottesville,VA; jgonzalez@virginia.edu

J Nucl Cardiol 2017;24:555-7.

$1071-3581 / \$ 34.00$

Copyright (C) 2016 American Society of Nuclear Cardiology. emerged as the major pharmacologic alternative to exercise stress. New ischemia-induced wall motion abnormalities constituted a positive test for ischemia. Sensitivity for CAD detection is higher, and specificity lower, for stress MPI compared to stress echocardiography. For this reason, stress echocardiography is commonly performed in patients at lower pre-test clinical risk for CAD. Conversely, with a higher sensitivity for CAD detection, stress MPI is advocated for higher risk patients with suspected or known CAD who need testing for appropriate clinical indications.

Over the past few decades, the percentage of patients referred for pharmacologic stress imaging, relative to exercise imaging, has been increasing from about $30 \%$ to more than $50 \%$ of stress imaging tests. ${ }^{2,3}$ In addition, a substantial number of patients initially referred for exercise stress are being triaged to pharmacologic stress. Patients who are unable to achieve target heart rate with exercise are changed over to predominantly vasodilator stress for the injection of tracer for radionuclide imaging, or dobutamine infusion for completion of stress echocardiographic testing. Currently, patients referred for PET MPI all undergo pharmacologic stress since the positron-emitting tracers (e.g., Rb-82) approved for clinical imaging have very short half-lives, thereby precluding upright exercise testing with its inherent delay in moving patients postexercise for imaging in the positron camera.

Because pharmacologic stress testing has been increasing over time, Argulian et $\mathrm{al}^{4}$ sought to determine the reasons for pharmacologic stress testing in consecutive patients referred for stress imaging at a single center. They found that $52 \%$ of patients in their hospital underwent initial pharmacologic stress imaging, despite the stated preference for symptom-limited exercise when feasible. In the study by Argulian et al, ${ }^{4}$ trained physicians triaged patients to either exercise or pharmacologic stress after reviewing clinical information, the resting ECG, and judging the ability to perform 
exercise. Patients with a recent myocardial infarction (MI), left bundle branch block, a diminution in LVEF, or resting wall motion abnormalities for echocardiography were directly referred for pharmacologic stress. Of the remaining patients, those who were either deemed too frail, deconditioned, unable to walk (e.g., orthopedic, neurologic, or vascular disease), or where safety concerns existed for exercise, underwent pharmacologic stress. Additionally, patients who were uncooperative or poorly motivated to exercise, or those unable to reach $\geq 85 \%$ of age-predicted MPHR when attempting to exercise, underwent pharmacologic stress. Interestingly, in the overall cohort, $64 \%$ were originally considered for exercise. An additional $16 \%$ of patients were moved to pharmacologic stress after initially chosen for exercise as the stressor. The most common reasons for choosing pharmacologic stress were deconditioning (28\%), inability to walk (23\%), and frailty (19\%). As expected, patients who underwent pharmacologic stress were older, heavier, and had a greater prevalence of prior revascularization, prior MI, CHF, diabetes, and hypertension than patients who underwent exercise stress imaging. This is consistent with the observation that patients undergoing pharmacologic stress have a higher annual cardiac death or nonfatal MI rate compared to patients undergoing exercise stress MPI. Navarre et al reported an annual hard event rate of $10.9 \%$ for pharmacologic MPI patients with severe perfusion defects, compared to $6.4 \%$ for exercise MPI patients with severe defects. ${ }^{5}$ Similar findings were reported by Hachamovitch et al in elderly patients. ${ }^{6}$ The annualized cardiac mortality was $7.8 \%$ for patients with moderately abnormal MPI results with pharmacologic stress vs $2.7 \%$ with such abnormal results in patients who underwent exercise MPI. Myers et al reported that exercise capacity was a stronger predictor of mortality than cardiovascular risk factors. ${ }^{7}$ Other prognostic variables derived from ExECG have proven useful for risk stratification. ${ }^{8}$

Some findings of the study by Argulian et $\mathrm{al}^{4}$ deserve mention and further discussion. Surprisingly, $81 \%(\mathrm{n}=922)$ of the patients in this cohort had stress echocardiography compared to only $19 \%(\mathrm{n}=218)$ who underwent SPECT MPI. Although the authors state that they "promulgate stress echocardiography as a preferred test in low likelihood of ischemia patients," inspection of Table 2 in the paper shows that patients referred for stress echocardiography had a $72 \%$ prevalence of hypertension, almost $40 \%$ had diabetes, $20 \%$ had prior revascularization, and $20 \%$ had either a prior MI or congestive heart failure. Thus, this patient population appeared somewhat skewed toward favoring stress echocardiography over stress MPI since many were not at low risk. Of the 922 patients who underwent stress echocardiography, $462(50.1 \%)$ had dobutamine administration. Another finding that warrants discussion is that of the 415 patients in the study who exercised to target heart rate, the mean metabolic equivalents (METS) achieved was $9.0 \pm 2.7$. It is likely that many of these patients might have been adequately risk stratified with ExECG alone, since a significant percentage surely achieved $\geq 10$ METS. Bourque et $\mathrm{al}^{9}$ found that in a patient population with an intermediate to high risk of CAD, those achieving $\geq 10$ METS had a markedly low prevalence of ischemia and very low rates of cardiac death $\left(0.1 \% \cdot\right.$ year $\left.^{-1}\right)$ and nonfatal MI $\left(0.7 \% \cdot\right.$ year $\left.^{-1}\right)$ during follow-up. ${ }^{10}$ Myocardial imaging did not provide incremental prognostic information in this group of patients with good exercise tolerance.

It appears that some patients referred directly for pharmacologic stress imaging can indeed complete a Bruce or Naughton protocol exercise test. In one study, $62 \%$ of patients referred for clinically indicated pharmacologic MPI actually were able to reach target heart rate with exercise alone. ${ }^{11}$ The authors of this study concluded that pharmacologic stress testing may be over-ordered in current clinical practice. Their study showed the feasibility of combining regadenoson with peak exercise in patients unable to achieve target heart rate during exercise testing. This approach permits those patients to achieve $\geq 85 \%$ of MPHR for age if they are able to do so, with regadenoson given only to those failing to achieve this endpoint. Partington et $\mathrm{al}^{12}$ reported similar findings in that $32 \%$ of patients in their study had submaximal heart rate responses during performance of an exercise MPI. Administering regadenoson in these patients at their submaximal HR was safe and allowed more accurate imaging data to be collected. Another protocol that is undertaken in our laboratory for patients unable to achieve target heart rate is to stop the exercise test prior to administering the imaging agent (e.g., sestamibi) and convert the patient to a resting regadenoson study. These approaches allow for reporting of both exercise tolerance, an excellent prognostic variable, and the results of imaging.

Pharmacologic stress with vasodilators is generally safe when patients are diligently monitored. However, some potential life-threatening side effects have been reported ${ }^{13}$ including rare cases of complete heart block and asystole. ${ }^{14}$ The risk of death or a MI during exercise testing is extremely low. Dobutamine carries the highest risk for adverse events of all the stress modalities. ${ }^{13}$ These include sustained ventricular tachycardia, ventricular fibrillation, asystole, acute MI, and atrial tachyarrhythmias. Serious adverse events range from 1/ 210 to $1 / 557 .^{13}$

The study by Argulian et $\mathrm{al}^{4}$ expands our knowledge of the reasons why such a high percentage of 
patients referred for stress imaging undergo pharmacologic stress rather than exercise stress, the preferred stress modality for optimal risk assessment. Patients undergoing pharmacologic stress echocardiography or MPI were older (mean age 65 years), had a higher BMI (mean 32.1), and a higher prevalence of a prior MI and heart failure. Many were frail, deconditioned, and unable to adequately ambulate on a treadmill for medical reasons. This is reflective of the overall higher risk status of their patient population. This high percentage of pharmacologic stress studies may not apply to other centers with lower risk patients referred for stress testing. Often, choosing the right test for the right patient is not easy in a busy stress laboratory, particularly in determining a patient's ability to adequately complete a treadmill test. Several questionnaires aimed at assessing a patient's ability to achieve a good exercise workload, like the Duke Activity Status Index (DASI), have been described. ${ }^{15}$ It appears that even trained clinicians and nurses tend to underestimate the ability of patients to exercise to their target heart rate. Furthermore, they may surmise that, with pharmacologic stress, imaging results will be more diagnostic for CAD detection and risk stratification. This also contributes to the growing percentage of patients undergoing initial pharmacologic testing. When the ability to adequately exercise is obvious, or even when it is uncertain, exercise should be the preferred starting stress modality. If the target exercise heart rate, or a high MET level is not achieved, then vasodilator stress MPI can be performed as described above. Alternatively, vasodilator PET MPI, with measurement of absolute blood flow and coronary flow reserve, provides even better separation of highand low-risk patients after imaging compared to just identifying relative defects in perfusion. ${ }^{16}$ Finally, it should be emphasized that many patients can be accurately evaluated solely with an exercise treadmill test without any imaging, and this avoids radiation exposure for radionuclide MPI and reduces the cost for either stress MPI or stress echocardiography.

\section{References}

1. Iskandrian AS, Heo J, Kong B, Lyons E. Effect of exercise level on the ability of thallium-201 tomographic imaging in detecting coronary artery disease: Analysis of 461 patients. J Am Coll Cardiol 1989;14:1477-86.

2. Rozanski A, Gransar H, Hayes SW, Min J, Friedman JD, Thomson LE, et al. Temporal trends in the frequency of inducible myocardial ischemia during cardiac stress testing: 1991 to 2009. J Am Coll Cardiol 2013;61:1054-65. doi:10.1016/j.jacc.2012.11.056.
3. Gharacholou SM, Pellikka PA. Trends in noninvasive testing for coronary artery disease: Less exercise, less information. Am J Med 2015;128:5-7. doi:10.1016/j.amjmed.2014.08.019.

4. Argulian E, Po JR, Uretsky S, Kommaraju K, Suketukumar P, Agarwal V, et al. Comparison of the current reasons for undergoing pharmacological stress during echocardiographic and radionuclide stress testing. $\mathrm{J}$ Nucl Cardiol 2016. doi: 10.1007/s12350-016-0398-2.

5. Navare SM, Mather JF, Shaw LJ, Fowler MS, Heller GV. Comparison of risk stratification with pharmacologic and exercise stress myocardial perfusion imaging: A meta-analysis. J Nucl Cardiol 2004;11:551-61.

6. Hachamovitch R, Kang X, Amanullah AM, Abidov A, Hayes SW, Friedman JD, et al. Prognostic implications of myocardial perfusion single-photon emission computed tomography in the elderly. Circulation 2009;120:2197-206. doi:10.1161/CIRCULATIONA HA.108.817387.

7. Myers J, Prakash M, Froelicher V, Do D, Partington S, Atwood JE. Exercise capacity and mortality among men referred for exercise testing. N Engl J Med 2002;346:793-801.

8. Bourque JM, Beller GA. Value of exercise ECG for risk stratification in suspected or known CAD in the era of advanced imaging technologies. JACC Cardiovasc Imaging 2015;8:1309-21. doi: 10.1016/j.jcmg.2015.09.006.

9. Bourque JM, Holland BH, Watson DD, Beller GA. Achieving an exercise workload of $>$ or $=10$ metabolic equivalents predicts a very low risk of inducible ischemia: Does myocardial perfusion imaging have a role? J Am Coll Cardiol 2009;54:538-45. doi: 10.1016/j.jacc.2009.04.042.

10. Bourque JM, Charlton GT, Holland BH, Belyea CM, Watson DD, Beller GA. Prognosis in patients achieving $\geq 10$ METS on exercise stress testing: Was SPECT imaging useful? J Nucl Cardiol 2011;18:230-7. doi:10.1007/s12350-010-9323-2.

11. Ross MI, Wu E, Wilkins JT, Gupta D, Shen S, Aulwes D, et al. Safety and feasibility of adjunctive regadenoson injection at peak exercise during exercise myocardial perfusion imaging: The Both Exercise and Regadenoson Stress Test (BERST) trial. J Nucl Cardiol 2013;20:197-204. doi:10.1007/s12350-013-9679-1.

12. Partington SL, Lanka V, Hainer J, Blankstein R, Skali H, Forman DE, Di Carli MF, Dorbala S. Safety and feasibility of regadenoson use for suboptimal heart rate response during symptom-limited standard Bruce exercise stress test. J Nucl Cardiol 2012;19:970-8. doi:10.1007/s12350-012-9562-5.

13. Dilsizian V, Gewirtz H, Paivanas N, Kitsiou AN, Hage FG, Crone NE, et al. Serious and potentially life threatening complications of cardiac stress testing: Physiological mechanisms and management strategies. J Nucl Cardiol 2015;22:1198-213. doi:10.1007/s12350015-0141-4.

14. Rosenblatt J, Mooney D, Dunn T, Cohen M. Asystole following regadenoson infusion in stable outpatients. J Nucl Cardiol 2014;21:862-8. doi:10.1007/s12350-014-9898-0.

15. Phillips L, Wang JW, Pfeffer B, Gianos E, Fisher D, Shaw LJ, et al. Clinical role of the Duke Activity Status Index in the selection of the optimal type of stress myocardial perfusion imaging study in patients with known or suspected ischemic heart disease. J Nucl Cardiol 2011;18:1015-20. doi:10.1007/s12350-011-9456-y.

16. Murthy VL, Naya M, Foster CR, Hainer J, Gaber M, Di Carli G, et al. Improved cardiac risk assessment with noninvasive measures of coronary flow reserve. Circulation 2011;124:2215-24. doi: 10.1161/CIRCULATIONAHA.111.050427. 